\title{
Una concepción integradora del aprendizaje humano
}

\author{
An integrating conception of human learning
}

Diego J. González Serra ${ }^{1}$

\begin{abstract}
RESUMEN
En el decurso histórico de la psicología científica han sido desarrolladas diferentes teorías del aprendizaje que son unilaterales pues abordan aspectos parciales, los cuales son concebidos de manera absoluta en detrimento de la consideración de otros. Sirva de ejemplo la contraposición entre las teorías de estímulo - respuesta, de un lado, y las teorías cognitivas o humanistas del otro. Por ello, los que somos partidarios del conocimiento más pleno y objetivo del psiquismo, consideramos necesario trabajar en pro de una teoría dialéctica, sintética o integradora, del aprendizaje que asimile críticamente, vuelva a elaborar e integre todo lo que la psicología mundial ha aportado en esta dirección. De acuerdo con el principio de la unidad dialéctica del análisis y la síntesis, esto no niega sino afirma la necesidad de teorías analíticas o parciales que profundicen, sin hiperbolizar ni caer en enfoques unilaterales, en la naturaleza de los distintos aspectos y momentos del aprendizaje humano y animal. Nuestra posición es dialéctica por cuanto nos esforzamos por un enfoque multilateral que enfatice el aprendizaje humano como un reflejo de su medio socio histórico y a la vez tenga en cuenta su función creadora de aquello que se aprende. Planteamos la necesidad de desarrollar y exponer una teoría psicológica del aprendizaje como una temática central de la psicología general y de la pedagógica, de gran importancia teórica y práctica. Esta es una tarea colectiva y a largo plazo. En el presente artículo avanzamos algunos criterios que podemos discutir, mantener, abandonar o perfeccionar.
\end{abstract}

\begin{abstract}
Throughout the history of scientific psychology, different learning theories have been developed. These theories are unilateral since they address partial aspects that are considered in a categorical way, namely, without considering different ones. The polarity between stimulus-response theories and cognitive and humanist theories is an example of this position. Hence, being supporters of a fullest and most objective knowledge of the psyche, we consider necessary to work for a dialectical, synthetical or integrating learning theory that would critically incorporate, re-elaborate and integrate everything that the worldwide psychology has provided related to this direction. According to the dialectical principle of analysis and synthesis unity, it is not denied, but affirmed, the need for analytical or partial theories that deepen the nature of different aspects and moments of human and animal learning, without hyperbolizing or falling into unilateral approaches. The position we assume is dialectical since we strive for a multilateral approach that emphasizes human learning as a reflex of the sociohistorical context, and, simultaneously, considers the creative function of what is learned. We advocate the need to develop and present a psychological learning theory as a pivotal theme for the general and the pedagogical psychology, given its theoretical and practical value. This is a collective and long-term task. In the present article, we advance some points that can be discussed, maintained, abandoned or improved.
\end{abstract}

\footnotetext{
${ }_{1}^{1}$ Profesor Titular Adjunto de la Carrera de Psicología de la Universidad de la Habana. Licenciado en Psicología (Universidad de la Habana, 1966). Post-graduación en Lovaina (Bélgica) y en Moscú (URSS). Profesor e investigador de la motivación humana en la Facultad de Psicología, la Academia de Ciencias y el Instituto Pedagógico E.J. Varona. Autor de 14 libros sobre problemas teóricos de la psicología, la motivación, psicopedagogía y el pensamiento psicológico de José Martí. Miembro de HONOR de la Cátedra Vygotski. Contacto: diegonza@infomed.sld.cu
} 
Palavras clave: Concepto de aprendizaje. Aprendizaje human. Formas y niveles del aprendizaje.
Keywords: Learning concept. Human learning. Learning methods and levels.

\section{El concepto de aprendizaje}

Entendemos por aprendizaje la génesis, transformación y desarrollo de la psiquis y del comportamiento que ella regula en función de la actividad, o sea, de la interacción del sujeto con su medio. Aquí empleamos el término actividad en su acepción más amplia que incluye tanto la actividad interna o psíquica como la externa y dentro de esta última la actividad con los objetos materiales y la comunicación.

La esencia del aprendizaje consiste en la aparición y modificación de los procesos psíquicos y del comportamiento tanto en una faceta o dimensión afectiva como cognoscitiva. Se aprenden no sólo hábitos, conocimientos, y habilidades, sino también actitudes, rasgos volitivos, emociones, sentimientos y necesidades. Por ello el aprendizaje se refiere tanto al proceso de enseñanza como al de educación.

Aunque es necesario reconocer la influencia de los determinantes biológicos o innatos, fruto de la herencia y de la maduración, el estudio del aprendizaje se centra en la investigación de lo adquirido en virtud de la experiencia del sujeto, de su interacción con el medio. En el animal es mayor la influencia de los determinantes innatos o biológicos. Sin embargo, la personalidad humana es fundamentalmente un producto del aprendizaje.

El término aprendizaje se refiere a los mismos hechos a que alude el principio psicológico del determinismo de lo psíquico en la unidad de la psiquis y la actividad como planteó S. L. Rubinstein, (1965, 1969) donde las causas externas actúan a través de las condiciones internas.

En la medida que el aprendizaje es una función especial de la escuela, la teoría sobre el mismo tiene una importancia cardinal para la psicología educacional y para la pedagogía. 


\section{La unidad de lo cognoscitivo y lo afectivo en la determinación del aprendizaje}

Todo lo que se aprende surge en virtud de la unidad inseparable de lo cognoscitivo y lo afectivo. Podríamos clasificar los determinantes inmediatos del aprendizaje en:

1- La estructuración psíquica que implica determinados procesos y reflejos o contenidos psíquicos, los cuales regulan la actividad y conducen a la satisfacción o insatisfacción de las necesidades del sujeto.

2- El reforzamiento que consiste en el componente afectivo positivo o negativo que fija o elimina esos procesos o reflejos psíquicos.

3- La reiteración o repetición en la actividad de ambos determinantes anteriores.

La estructuración psíquica puede ser preponderantemente cognoscitiva o afectiva. Puede ser la estructura cognitiva del campo perceptivo o del pensamiento en la solución de problemas. Puede ser la estructura afectiva, el sistema de tensiones o valencias del campo perceptivo o la serie de medios - fines del proceso volitivo, etc.

La estructura psíquica cognoscitiva produce fundamentalmente la regulación ejecutora que según S. L. Rubinstein (1965, p. 396 - 397) se generaliza y automatiza para dar lugar a los hábitos, conocimientos, habilidades y capacidades. Pues bien, esta generalización y automatización se produce en virtud del reforzamiento.

La estructura psíquica afectiva participa fundamentalmente en la regulación inductora o motivación que se generaliza y automatiza para dar lugar al carácter. Pues bien, esto ocurre en virtud del reforzamiento. De esta manera el aprendizaje engendra la personalidad humana.

El término reforzamiento tiene un marcado sabor conductista y se refiere por lo general a un estímulo o situación externa que refuerza las conexiones. Aquí le damos a este término una acepción totalmente diferente. Por reforzamiento entendemos el componente o aspecto afectivo interno, agradable o desagradable, 
que es el que realmente refuerza, debilita o inhibe la estructuración psíquica y la convierte o no en una propiedad o formación psíquica estable de la personalidad. También debemos considerar la ausencia de reforzamiento.

El reforzamiento puede ser positivo o negativo. El reforzamiento positivo consiste en un proceso afectivo positivo, agradable, que fija, conserva, graba, o consolida aquellos procesos y reflejos psíquicos que conducen a la satisfacción de la necesidad o se asocian a ella.

El reforzamiento negativo consiste en un proceso afectivo negativo, desagradable, que inhibe o evita aquellos procesos y reflejos psíquicos que conducen a la insatisfacción de las necesidades o se asocian a ella.

En esencia, el aprendizaje se produce cuando una determinada estructuración psíquica engendra procesos y reflejos que conducen a la satisfacción o insatisfacción de las necesidades o a la ausencia de ambas y en consecuencia, en virtud de ese reforzamiento o falta de reforzamiento afectivo, dichos procesos y reflejos psíquicos se fijan, se consolidan o por el contrario se debilitan, finalmente desaparecen o son inhibidos o evitados.

Este punto de vista supera la contraposición entre teóricos como Thorndike y Hull ${ }^{2}$, que destacan unilateralmente la importancia del reforzamiento y teóricos como Kohler y Koffka ${ }^{3}$ que enfatizan sólo la estructuración psíquica.

De acuerdo con su origen o fuente la estructuración psíquica puede ser material, comunicativa o interna. Es Material, cuando la estructuración psíquica refleja los objetos materiales que entran en interacción directamente con el sujeto. Estos procesos y reflejos psíquicos se dan al nivel sensorial y en la regulación psíquica de los movimientos musculares. La segunda fuente es la Comunicativa, cuando la estructuración psíquica surge a partir de la comunicación emocional o simbólica con otros sujetos. Por último, la Interna, es cuando la estructuración psíquica surge a partir de la propia actividad psíquica

\footnotetext{
2 E,Thorndike (1874-1946) psicólogo norteamericano creador del conexionismo quien destacó el aprendizaje por ensayo y error basado en la satisfacción.. C. L. Hull (1884.1952) psicólogo neo conductista norteamericano quien destacó como reforzador la reducción de la carencia biológica.

3 W. Köhler (1887-1967) y K. Koffka (1886-1941) psicólogos gestaltistas alemanes, de orientación fenomenológica, que basan el aprendizaje en la estructura cognoscitiva del campo psicológico.
} 
interna del sujeto que aprende de manera relativamente independiente de los estímulos externos. Puede ser una reestructuración en el plano de la percepción, una actividad imaginativa o del pensamiento o una planificación volitiva o la persistencia en una meta. En estos casos el proceso y reflejo psíquico que se aprende no procede directamente de un objeto material externo, ni de la comunicación con otros sujetos, sino que constituyen una creación del propio individuo que aprende.

Es importante considerar la diferencia entre los niveles animal y humano en que se produce la estructuración psíquica. En el animal y también en los niveles inferiores del ser humano, la estructuración psíquica opera a nivel sensorial, en la regulación psíquica de los movimientos musculares y en las reacciones emocionales, a nivel del primer sistema de señales. De manera específica en el hombre la estructuración psíquica actúa en la imaginación, el pensamiento, en la voluntad y el sentimiento, o sea, al nivel de la palabra. Así, la estructuración psíquica puede ser sensomotriz o verbal y a su vez, tanto la una como la otra pueden ser materiales, comunicativas o internas.

En el caso del reforzamiento este también puede tener diferentes fuentes: material, comunicativa o interna.

La fuente Material, es cuando la reacción afectiva es producida por un objeto material externo que afecta positiva o negativamente y de manera directa las necesidades y metas del sujeto que aprende.

La Comunicativa, ocurre cuando la reacción afectiva es producida por los éxitos o fracasos de otro sujeto al cual observa y con el cual se identifica el sujeto que aprende. En este caso la comunicación se produce por identificación con otro sujeto cuyos éxitos y fracasos engendran reacciones afectivas agradables y desagradables en el sujeto que aprende. Hablamos aquí de un reforzamiento vicariante. También el reforzamiento es comunicativo cuando la reacción afectiva es producida por la comunicación emocional o verbal. Por ejemplo, cuando el padre acaricia a su hijo o cuando el profesor le otorga una buena calificación. Por último, la Interna, cuando la afectividad es producida por procesos y reflejos psíquicos elaborados por el propio sujeto de manera relativamente independiente 
de los estímulos externos. Por ejemplo, la reestructuración en el plano de la percepción o el hallazgo de la imaginación o el pensamiento, que resuelven problemas y afirman o niegan perspectivas de satisfacción, la reestructuración volitiva, la auto valoración etc. producen un reforzamiento interno puesto que engendran vivencias afectivas agradables o desagradables.

Es importante considerar la diferencia entre los niveles animal y humano en que se produce el reforzamiento. En el animal (y también en el nivel inferior del ser humano) la necesidad actúa de manera directa y la reacción emocional se corresponde con el objeto externo. De manera específica en el hombre la necesidad es mediada por la conciencia, en el fin o proyecto, en los valores y convicciones del sujeto y aquí el proceso afectivo se experimenta como algo interno, vinculado a los proyectos, a los valores, a las convicciones y auto valoración del sujeto. Así, el reforzamiento puede ser sensomotriz o verbal y tanto el uno como el otro pueden ser materiales, comunicativos o internos.

\section{Determinación externa y autodeterminación en el aprendizaje}

Cabe destacar que las diferencias que acabamos de establecer entre los distintos tipos de estructuración psíquica y de reforzamiento ya anuncian dos tipos de aprendizaje: el interno y el externo.

El aprendizaje externo, o predominantemente externo es producido por la interiorización de determinantes del medio físico y social, los cuales actúan a través del psiquismo y de los procesos internos del sujeto para producirlo. El contenido que se va a aprender viene de fuera, es dado u ofrecido al sujeto.

El aprendizaje externo puede a su vez subdividirse en material y comunicativo. Si el sujeto entra en interacción con objetos materiales externos y aprende a acercarse a ellos o evitarlos, a utilizarlos o transformarlos en función de sus necesidades, se trata de un aprendizaje externo material. Si el sujeto se comunica con otros individuos y aprende a través de ese vínculo emocional, simbólico y de identificación, entonces se trata de un aprendizaje externo comunicativo. Muy a menudo el aprendizaje externo es material y comunicativo 
simultáneamente.

El aprendizaje interno o predominantemente interno es producido por la actividad psíquica del sujeto con una relativa independencia de los determinantes externos, pero parte de la experiencia anterior formada por la interiorización de lo externo y siempre actúa en el contexto del reflejo psíquico de las circunstancias actuales externas. Véase, por ejemplo, la reestructuración súbita en la percepción que encontró Köhler en los monos y la actividad del pensamiento, la reflexión volitiva, la elaboración de metas y la auto valoración, en el hombre. En el aprendizaje interno el contenido que se va a aprender es descubierto o creado por el propio sujeto.

El aprendizaje externo opera principalmente en virtud de la estructuración psíquica y del reforzamiento que denominamos material y comunicativo. El aprendizaje interno ocurre fundamentalmente a través de lo que hemos llamado estructuración psíquica y reforzamiento internos.

Además, debemos considerar un tipo de aprendizaje que constituye una síntesis superior de los dos anteriores: el aprendizaje interno - externo. Aquí el rol activo y creador del sujeto (el aprendizaje interno) conduce al externo y a su vez éste último repercute sobre el primero. Se trata, por ejemplo, en el ser humano, del rol activo del sujeto en la elaboración interna de una idea o hipótesis y en la búsqueda de información o verificación externa que la fundamente o compruebe; o en la elaboración de un ideal de sí mismo que conduce a someterse a situaciones externas que modifiquen su personalidad en la dirección deseada. En tales casos el sujeto asume una posición activa y creadora (he aquí el componente interno) pero se proyecta hacia la adquisición de un reflejo del medio (he aquí el componente externo).

Teóricos como Watson ${ }^{4}$ y seguidores han estudiado de manera unilateral y reduccionista el aprendizaje externo material. Por el contrario, los gestaltistas y humanistas destacaron sólo el aprendizaje interno. Y en consecuencia, ninguna de estas escuelas se planteó la unidad dialéctica que existe entre el aprendizaje externo y el interno. A continuación, intentaremos aproximarnos a la conducta, de la interacción objetiva estímulo - respuesta. 
comprensión teórica de esta unidad dialéctica.

En primer lugar, es necesario considerar cómo ambos tipos de aprendizaje se contienen recíprocamente. Todo aprendizaje, ya sea externo o interno, aunque es un producto inmediato o directo de los procesos psíquicos, es el resultado de la unidad de la actividad externa e interna del sujeto y sólo puede darse en la unidad y penetración de ambas.

En segundo lugar, es necesario destacar la determinación recíproca del aprendizaje externo e interno. El aprendizaje externo ocurre primero y crea las condiciones psíquicas para que pueda darse el aprendizaje interno. El pensamiento manual o concreto estudiado por Pavlov 5 en los monos, el cual constituye un aprendizaje interno, surge en virtud de que previamente el mono ha formado hábitos y conexiones sensomotrices en el decurso de la actividad externa y emplea estos hábitos en función de la reestructuración súbita de la percepción que lo lleva a la solución del problema. Las teorías cognitivistas y humanistas tienen el defecto fundamental de que no aprecian debidamente el condicionamiento externo del aprendizaje interno.

También el aprendizaje interno repercute sobre el externo. Por ejemplo, en el ser humano, el proceso del pensamiento que lleva a nuevas conclusiones teóricas lo conduce a experimentos u observaciones externas de una naturaleza superior y a la formación en la actividad externa de nuevos hábitos y habilidades más complejas y este es el caso del aprendizaje interno - externo que a un nivel sensomotriz y verbal es típico del ser humano. Claro, los teóricos estímulo respuesta no tienen en cuenta esto porque no reconocen el aprendizaje interno.

El aprendizaje externo y el interno existen en los animales y en el hombre. Los teóricos estímulo - respuesta estudiaron el aprendizaje externo en la ratas y Tolman dice que el aprendizaje por discernimiento (que es interno) también se da en la rata. Köhler estudió en los monos el aprendizaje interno, por discernimiento o insight. Sin embargo, mientras en los animales el aprendizaje interno se limita a la actividad sensorial y emocional, en el hombre se amplía cualitativamente con

\footnotetext{
${ }^{5}$ I. P. Pavlov (1849-1936) fisiólogo ruso, iniciador de la corriente reflexológica que destaca el estudio del funcionamiento físiológico del cerebro como fundamento de la psicología.
} 
la actividad del pensamiento, la imaginación, la voluntad y los sentimientos. Estos hechos requieren la consideración de los niveles del aprendizaje.

\section{Los niveles del aprendizaje}

Teniendo en cuenta el desarrollo de las especies animales que condujo al hombre y el desarrollo del individuo humano de recién nacido a persona adulta, es necesario señalar dos importantes niveles del aprendizaje: el sensomotriz y el verbal.

El aprendizaje sensomotriz es común al hombre y a los animales. El aprendizaje verbal es específico o propio sólo del ser humano.

El aprendizaje sensomotriz puede ser externo o interno, pero en ambos casos ocurre en virtud del primer sistema de señales, o sea, de los objetos y sujetos que actúan como señales y no como símbolos, ya sea como estímulos externos o como contenidos de la percepción y de la reacción emocional e impulsiva del sujeto.

El aprendizaje sensomotriz es el resultado de la experiencia individual del sujeto que aprende y engendra, de manera inconsciente, los hábitos y las costumbres.

El aprendizaje verbal puede ser externo o interno, pero en ambos casos ocurre en virtud de la asimilación de la palabra u otro tipo de actividad simbólica, ya sea como estímulos externos o como contenido de la conciencia humana. El aprendizaje verbal aporta al individuo la experiencia social, la cultura de la sociedad elaborada históricamente y engendra por lo general de manera consciente los conocimientos, habilidades y capacidades, las intenciones, proyectos, sentimientos y necesidades superiores, o sea, produce el psiquismo específicamente humano, la personalidad.

Lo típico del aprendizaje infantil humano en sus primeras etapas es la unidad indisoluble del aprendizaje sensomotriz con el verbal, pero predomina el sensomotriz. El proceso de enseñanza y educación consiste en el paso del predominio del aprendizaje sensomotriz (en el niño pequeño) al predominio del 
aprendizaje verbal que se aprecia ya desde la edad escolar y asume su máxima expresión en la personalidad adulta.

Por ello, en la formación del psiquismo humano, el aprendizaje verbal tiene la mayor importancia y sin embargo está basado en el aprendizaje sensomotriz, de manera directa en la primera infancia y en última instancia en el adulto.

La unidad indisoluble de ambas formas de aprendizaje se observa en su determinación recíproca. El aprendizaje verbal, en sus primeras etapas, surge en virtud del sensomotriz pues las palabras sólo pueden ser asimiladas en dependencia de su interconexión con los objetos materiales que entran en interacción con el sujeto. Pero una vez asimilado el lenguaje, el aprendizaje verbal adquiere una relativa independencia y sólo en última instancia es determinado, confirmado o rechazado, por el aprendizaje sensomotriz cuando el sujeto constata que sus ideas y proyectos se corresponden o no con los hechos materiales.

A su vez el aprendizaje sensomotriz es cada vez más determinado por el verbal. El reflejo dado en la percepción de los objetos materiales es mediado y modificado por las palabras que ha asimilado el sujeto. El psicólogo soviético L. S. Vygotski (1896-1934), iniciador de la escuela histórico cultural, destacó cómo la enseñanza escolar impulsa el desarrollo psíquico en el niño.

Esta determinación recíproca explica la penetración mutua de ambos niveles del aprendizaje. En el ser humano el aprendizaje sensomotriz está estructurado por la palabra y el verbal contiene al sensomotriz y se basa en él. Además, es imprescindible destacar que el aprendizaje sensomotriz en el hombre supone la interacción con los objetos de la cultura material y con las demás personas, lo cual crea las bases fundamentales para que se pueda producir el aprendizaje verbal.

En virtud de que la sensomotricidad en el hombre es guiada por la palabra, se estructura a un nivel superior por las complejas y esenciales relaciones que conlleva la palabra. Pero el aprendizaje sensomotriz no refleja en sí mismo la esencia de la realidad sino las conexiones externas entre estímulos y respuestas, y tiene un carácter reactivo, o sea, no opera independientemente de los estímulos 
externos ni de las sensaciones orgánicas y propioceptivas.

En virtud de que la palabra es además un estímulo del primer sistema (un sonido, un gráfico) puede participar también en el aprendizaje sensomotriz y ser un resultado de este aprendizaje. Por ello no existe aprendizaje verbal si no contiene en si mismo el aprendizaje sensomotriz. De esta forma pueden darse automatismos en el pensamiento verbal (los hábitos mentales) y los procesos y propiedades superiores son resultados de la automatización. Pero la palabra refleja en sí misma la esencia de la realidad y tiene un carácter activo, o sea, una relativa autonomía, pues puede movilizar al organismo independientemente de los estímulos externos y de las sensaciones orgánicas. De esta manera se aprecia la penetración recíproca y las diferencias esenciales entre ambas formas de aprendizaje.

Los teóricos estímulo - respuesta redujeron el aprendizaje humano a las características del sensomotriz externo y material y en consecuencia lo aislaron de todas las otras formas. Por el contrario, los teóricos humanistas enfatizaron la naturaleza específica del aprendizaje verbal interno pero no consideraron su unidad con otras formas inferiores y externas. Así, ambas posiciones se enfrentaron de manera absoluta perdiendo de vista la unidad del aprendizaje sensomotriz y el verbal. Corresponde a Lev Vygotski y sus seguidores el mérito de haber enfatizado dentro de la psicología soviética el aprendizaje verbal externo en unidad con el sensomotriz.

Así, el aprendizaje humano presenta simultáneamente los siguientes niveles y formas en unidad dialéctica:

\begin{tabular}{|l|c|c|}
\hline & & Externo \\
\hline & Sensomotriz & Interno \\
\hline & & \\
\hline Aprendizaje humano & & \\
\hline & & Externo \\
\hline & & Interno \\
\hline
\end{tabular}


Cada una de estas formas o niveles opera en el contexto de las demás y es necesario explicarlo en su naturaleza y evolución por dicho contexto.

La aceptación de la existencia del aprendizaje sensomotriz en el hombre indica la posibilidad de trasladar al ser humano los resultados obtenidos en investigaciones con animales. Sin embargo, no puede olvidarse que el aprendizaje sensomotriz es verbal en el hombre y opera de manera diferente en el contexto de los niveles superiores y específicos de éste.

\section{La interrelación jerárquica de las diferentes formas y niveles del aprendizaje}

Ya hemos señalado, primeramente, la unidad dialéctica del aprendizaje externo con el interno y, posteriormente, la del aprendizaje sensomotriz y el verbal. Falta ahora responder la pregunta: ¿Cómo se relacionan todas estas formas de aprendizaje en la determinación del psiquismo humano?

Diversos intentos se han hecho en esta dirección. (Véase, por ejemplo, Gagné, R.M.,1971; Petrovski, A. V., 1980 A). Aunque todas las formas de aprendizaje se encuentran en unidad simultánea en el hombre adulto, si fuéramos a considerar cuáles surgen o predominan primero y cuáles después en el desarrollo del ser humano, podríamos plantear la siguiente secuencia:

1. El aprendizaje externo sensomotriz

2. El aprendizaje interno sensomotriz

3. El aprendizaje externo verbal

4. El aprendizaje interno verbal

5. La integración de todas las formas del aprendizaje bajo el predominio del aprendizaje interno verbal, lo cual ocurre en el adulto.

En esta secuencia las primeras formas crean la base para la aparición de las superiores y las determinan y a su vez, al surgir estas últimas repercuten sobre las primeras, elevándolas a un nivel superior. En consecuencia, el aprendizaje interno verbal es el nivel superior y el determinante fundamental de 
que la personalidad alcance su desarrollo más armónico y completo, pero a su vez depende del aprendizaje externo verbal y del sensomotriz y es determinado por ellos. Por lo tanto, todas las formas del aprendizaje deben ser desarrolladas armónicamente en la búsqueda del despliegue máximo del aprendizaje interno verbal en su interdependencia con el externo verbal y el sensomotriz.

Sin embargo, el grado de desarrollo social alcanzado por la humanidad hasta el presente limita a ciertas minorías el aprendizaje interno verbal y establece de manera predominante en la escuela el aprendizaje externo verbal. Esta es la enseñanza tradicional que Piaget, Rogers (1982) y Davidov (1986) quieren superar. Pensamos que la realización del ideal humanista supone el logro del desarrollo masivo del aprendizaje interno verbal en unidad con el externo verbal y el sensomotriz.

\section{Conclusiones}

Hasta aquí nuestras consideraciones teóricas y a partir de ellas, ¿qué recomendaciones podemos hacer a nuestros pedagogos?

1- Si el aprendizaje es la resultante de la unidad de lo cognitivo y lo afectivo y de su reiteración en la actividad, debemos ocuparnos tanto de la estructuración cognoscitiva y motivacional que conduzca a nuevos reflejos psíquicos como del proceso afectivo que los consolide. Es necesario unir dialécticamente la enseñanza y la educación, el conocimiento y el afecto.

2- Si el aprendizaje resulta de la unidad de lo sensomotriz y lo verbal es necesario combinar la enseñanza teórica con la práctica, con la observación de los hechos a los que alude la palabra. En la medida en que el aprendizaje verbal se apoye más firmemente en los hechos, en la experiencia sensorial y en la práctica, se consolidará y desarrollará de la mejor manera.

3- Si el aprendizaje resulta de la unidad de la actividad externa y la interna, es necesario comenzar por la interiorización, por la asimilación 
de los conocimientos y combinarla con métodos activos que promuevan el aprendizaje interno, o sea, la participación activa del sujeto en la creación de sus propios conocimientos, proyectos y actitudes, y de aquí volver a la asimilación activa de nuevos contenidos externos.

Es necesario, de inmediato, eliminar este tipo de enseñanza fría, sin emoción ni motivación, puramente verbal y reproductiva, alejada de la práctica y que no promueve el rol activo y creador del sujeto que aprende. Esto resulta decisivo para crear al ser humano que necesitamos y con el que soñó José Martí6.

\section{Referências}

DAVIDOV, V.V. (). Los Principios de la Enseñanza en la Escuela del Futuro. In: Iliasov y Liaudis. Antología de la psicología pedagógica y de las edades. Editorial Pueblo y Educación. La Habana, 1986.

GAGNÉ, R.M. Las condiciones del aprendizaje. Editorial Aguilar, Madrid, 1971.

GONZÁLEZ, D.J. Marti y la ciencia del espíritu. Editorial Si - mar S.A. La Habana, 1999.

GONZÁLEZ, D.J. Martí e a psicologia. Editorial Escrituras. São Paulo, Brasil, 2001.

PETROVSKI, A.V. Psicología evolutiva y pedagógica. Editorial Progreso. Moscú, 1980a.

PETROVSKI, A.V. Psicología general. Editorial Progreso. Moscú, 1980b.

PIAGET, Jean. Psicología de la inteligencia. Editorial Psique. Buenos Aires, 1955.

ROGERS, C. R. Libertad y creatividad en la educación. Ediciones Paidos, Barcelona - Buenos Aires, 1982.

RUBINSTEIN, S.L. El ser y la conciencia. Editorial Nacional de Cuba. Editora Universitaria. La Habana, 1965.

\footnotetext{
${ }^{6}$ José Martí (1853-1895) héroe nacional cubano, impulsor de la guerra de independencia que liberó a Cuba del dominio colonial español y que dejó un profundo y multifacético pensamiento político, filosófico, pedagógico y psicológico.
} 
RUBINSTEIN, S.L. Principios de psicología general. Edición Revolucionaria. La Habana, 1969.

VIGOTSKI, L. Pensamiento y lenguaje. Edición Revolucionaria. La Habana, 1981.

VIGOTSKI, L. Historia del desarrollo de las funciones psíquicas superiores. Editorial Científico Técnica. Ciudad de la Habana, 1982.

Recebimento: 31.07 .18

Aprovação: 19.08.18 